\title{
Design of automatic obstacle avoidance Car Based on STM32
}

\author{
Huang Jian \\ XiJing University, Xi'an 710123, China; \\ 565200245@qq.com
}

Keywords: Automatic tracking; photoelectric sensor; automatic obstacle avoidance

Abstract: In modern industrial production and life, the automobile has become an important means of transportation. However, with the increase in the number of cars, traffic accidents are also increasing. Therefore, the requirements of the performance of the car is also getting higher and higher, and intelligent will be an important development direction of the car in the future. Obstacle avoidance, automatic tracking driving will be the focus of the study. This paper is to study how to realize the automatic tracking and obstacle avoidance, the photoelectric sensor, according to the reflected light intensity, the black and white line tracking. Ultrasonic ranging is used to avoid obstacles. The test results show that the design achieves the expected goal, realize the automatic tracking and obstacle avoidance, has a certain practical value.

\section{Introduction}

In the various electronic design competitions and training of college students in innovation and entrepreneurship, there have been a variety of cars, from the original automatic round-trip car to automatic obstacle avoidance car, remote control car, smart car and so on. The requirements and functions of the car are also increasing. These designs can not only train students' practical ability, but also test students' ability of designing hardware circuits and software programming ability. What is more important is to improve students' ability to analyze and solve problems. Laid a certain foundation for the future work.

\section{Specific hardware circuit design。}

\section{Tracking circuit design}

The tracking circuit shown in figure 1. In Figure 1, the power supply is powered by $3.3 \mathrm{~V}$, and the photoelectric sensor adopts ST188. The sensor is a transmitter and receiver, and the 1 and 2 ends of the figure are connected with a photoelectric diode to adjust the resistance of the R68, so that the light intensity can be changed. The 3 and 4 terminals are connected with a phototransistor, and when the light is shining, the triode is connected and the T2 outputs are low. When the reflected light is very weak, the triode is cut off and the T2 output is high. According to this principle, can realize the automatic tracking of black and white line. 


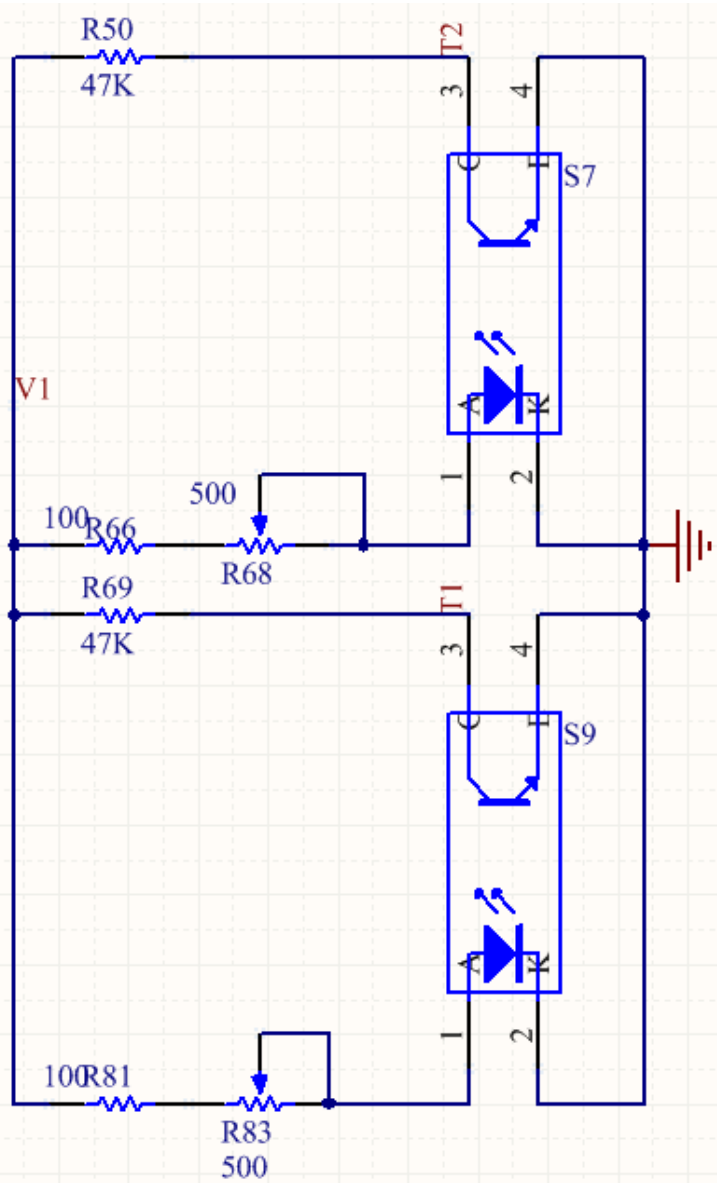

Figure 1 photoelectric sensor circuit diagram

Figure 2 shows the ultrasonic emission circuit diagram, the PWM wave generated by the microcontroller, $40 \mathrm{KHz}$ frequency, access to CD4096, and the signal conditioning, and launch it.

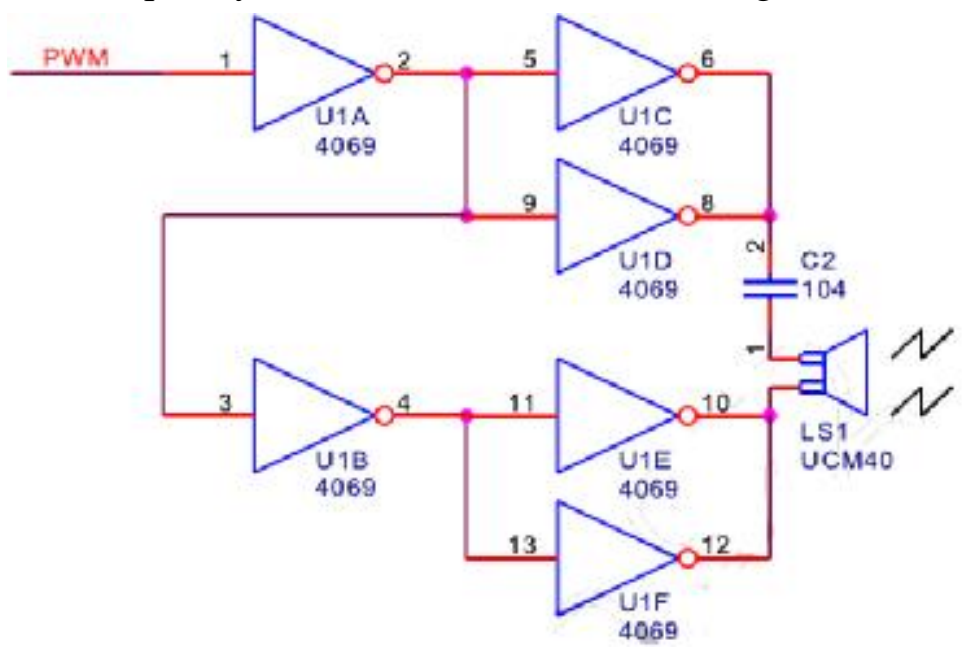

Fig. 2 ultrasonic emission circuit diagram

\section{Software programming}

In this design, under KEIL 5, programming with $C$ language. The initialization of ultrasonic wave is completed and the distance measurement result is calculated.

void USART3_IRQHandler(void)

\{

if(USART3->SR \& $(1<<5))$ 


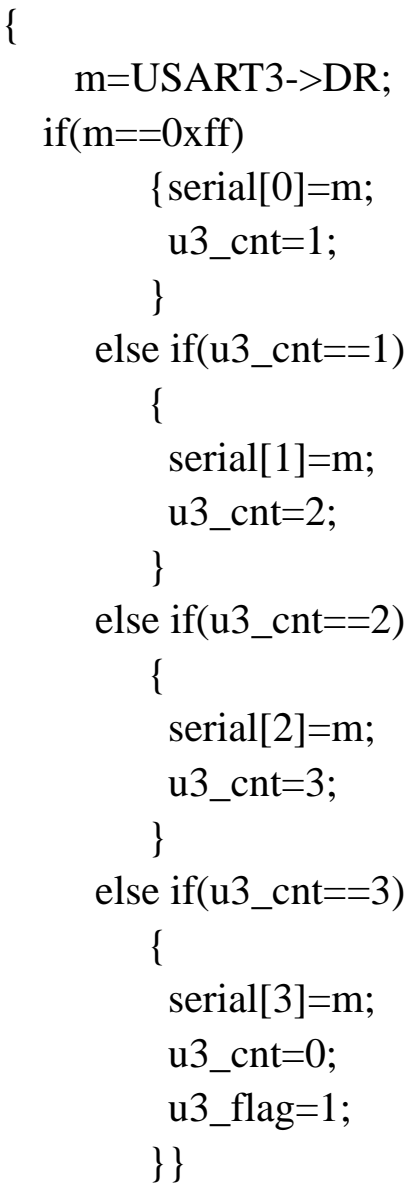

The above code implements the reception of ultrasonic measurement data. void Count(void)

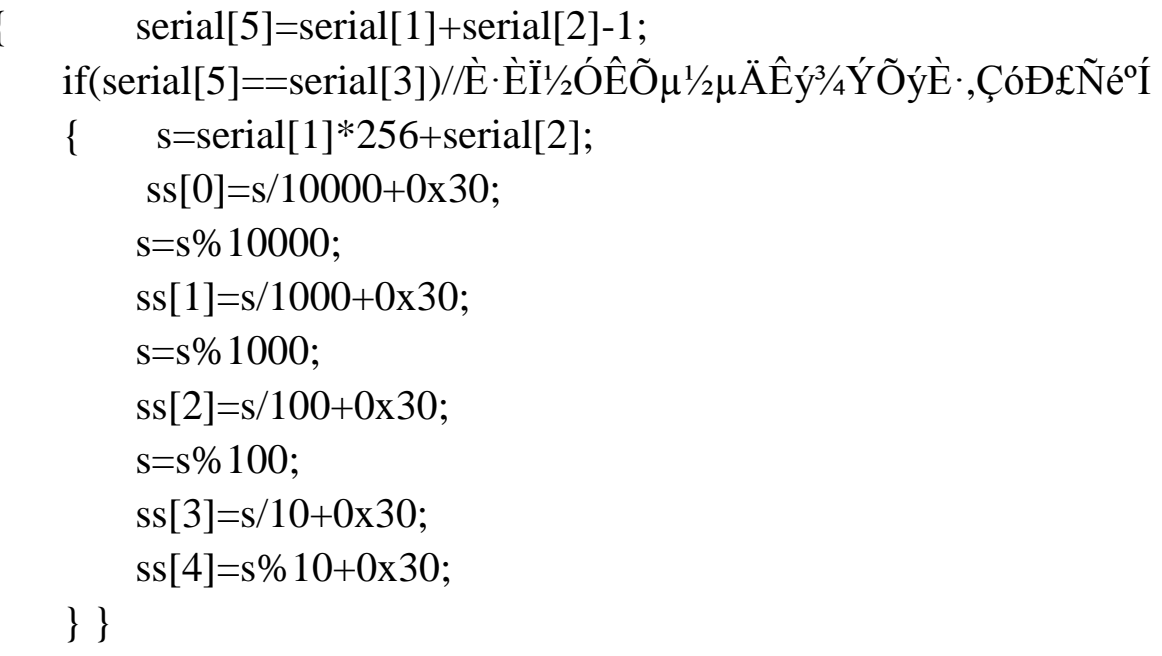

The above code implements the calculation of distance.

\section{Summary}

This paper expounds the realization of the black and white line tracking using photoelectric sensor for obstacle avoidance using ultrasonic ranging, hardware circuit design is given, its working principle is described. In KEIL MDK using $C$ language program, to achieve the control of tracking and obstacle avoidance, to achieve the desired purpose. It has certain practical value. 


\section{Reference}

[1] Lv Yunfang, Chen Shuaishuai, Hao Xingsen, Zhang Hao. The design and implementation of [J] intelligent tracking car C51 advanced language program based on the control of laboratory research and exploration,.2015,34 (3): 142-145.

[2] Liao Ping, Wu Yuesong, Wang Peng, Yue, Lili. Ultrasonic ranging system based on CAN bus, $[\mathrm{J}]$, instrument technology and sensor.2014,10:62-65

[3] Yao Yongping.STC12C5A60S2 series microcontroller manual [M], Acer crystal technology, 2011,8-9

[4] Tang Sichao. Actual design of embedded system software -- Based on IAR Embedded Workbench [M].: Beihang University press, 2010

[5] Guo Qing. Design of ultrasonic ranging collision avoidance system based on [J], STC89C52, instrument technology and sensor.2011,6:74-77 